\title{
Analytical Considerations of the Flow Boiling Heat Transfer in Metal-foam Tubes
}

\author{
Y.P Du ${ }^{1}$, C.Y. Zhao ${ }^{* 1,2}$, Y. Tian $^{2}$, Z.G. Qu ${ }^{1}$ \\ ${ }^{1}$ School of Energy and Power Engineering, Xi' an Jiaotong University, 710049, China \\ ${ }^{2}$ School of Engineering, University of Warwick, CV4 7AL, United Kingdom \\ *Corresponding author, Email: C.Y.Zhao@warwick.ac.uk \\ Tel: +44 (0)2476522339, Fax: +44 (0)24 76418922
}

\begin{abstract}
A newly simplified microstructure model of metal foams was carried out for flow boiling heat transfer in metal-foam filled tube. Fin analysis, heat transfer network and superposition correlation were adopted to obtain the equivalent heat transfer coefficient for flow boiling. Based on annular pattern, fluid can be divided into vapor region in the center of the tube and liquid region near the wall. However, it was assumed that nucleate boiling performed only in the liquid region. The analytical solution was verified by its good agreement with experimental data. The parametric study on heat transfer coefficient and boiling mechanism were also carried out.
\end{abstract}

Keywords Metal foams; Flow boiling; Microstructure model; Analytical solution

Published in: Heat and Mass Transfer 48(1), 2012, pp. 165-173.

Doi: $\quad$ doi: $10.1007 / \mathrm{s} 00231-011-0853-1$

Cited as: $\quad$ Y.P. Du, C.Y. Zhao, Y. Tian, Z.G. Qu, "Analytical Considerations of Flow Boiling Heat Transfer in Metal-foam Filled Tubes", Heat and Mass Transfer 48(1), 2012, pp. 165-173. 


\section{Introduction}

Saturated nucleate flow boiling has been extensively investigated [1-3]. Rohsenw [1] used an assumption of superposition to consider both nucleate and convective boiling effects on the flow boiling heat transfer coefficient. Dengler and Addoms [2] evaluated the equation for the two-phase heat transfer coefficient except for the nucleation. Later on, Chen [3] proposed a new correlation covering both the region of forced convection and nucleate boiling, which was proved consistent with experimental data by other researchers. However, for open-cell metal foams, flow boiling mechanism in metal foams is different and complicated due to the complexity of solid matrix. The open-cell metal foams have unique structures and high porosities (usually bigger than $85 \%$ ), so the results of the previous studies for the boiling heat transfer in porous media (usually the packed bed of particles with relatively low porosities less than $45 \%$ ) can not be applied mechanically to the case for metal foams.

It is evident that the characteristics of the flow boiling heat transfer in metal foams are strongly dependent on the metal foam structures. Mo [4] established an analytical model for open-cell metal foams, containing two kinds of structures, called V-type and H-type, in order to describe the microstructures of metal foams for simplification. The cylinder diameter and specific surface area of metal foams obtained by them were consistent with previous studies. Furthermore, good agreement for heat transfer coefficient was achieved between analytical results and experimental data. However, in these two models the first-class boundary conditions were presumed in which wall temperature was set constant. Krishnan [5,6] carried out a single unit cell structure to simulate heat transfer in open-cell metal foams. The void was assumed to be spherical whilst pores were located at the vertices. The total heat transfer coefficient by this model was highly accordant with experimental data. Boomsma et al. [7] defined an innovative microstructure of metal foams and then developed a special method for simulating flow and heat transfer in open-cell metal foams. The pressure loss and velocity distribution in foam-filled channels were investigated, which were 25\% lower than those of experiment. However, these s models were established only for single phase flow conditions, and flow boiling characteristics with metal foams were not involved yet.

Murthy et al. [8] established a semi-analytical model of the bubble dynamics to predict heat transfer performance of flow boiling on porous structured surfaces. The predicted heat flux by them had 30\% deviation compared with experimental results. Wang and Cheng [9] developed a multiphase model in porous media in which the exact solutions were provided. However, in their model only one-dimensional problem was considered. Zhao et al $[10,11]$ experimentally and numerically investigated flow boiling heat transfer in metal-foam filled tubes based on wavy flow and annular flow. Non-equilibrium effects were considered for establishing two phase equations. The numerical results agreed well with experimental data. However, the governing equations were only valid for single phase flow rather than multiphase flow situation. In addition, all of the 
studies by them neglected the microstructure of metal foams, which is quite important for boiling heat transfer analysis.

In this paper, a newly simplified microstructure model of metal foams was carried out. Based on the model, an exact analytical solution for flow boiling heat transfer in metal-foam filled tube was carried out. The analytical solutions for heat transfer coefficient of flow boiling under annular flow pattern were precisely formulated. The predicted results agreed well with previous experimental results.

\section{Simplified model for microstructure of metal foams}

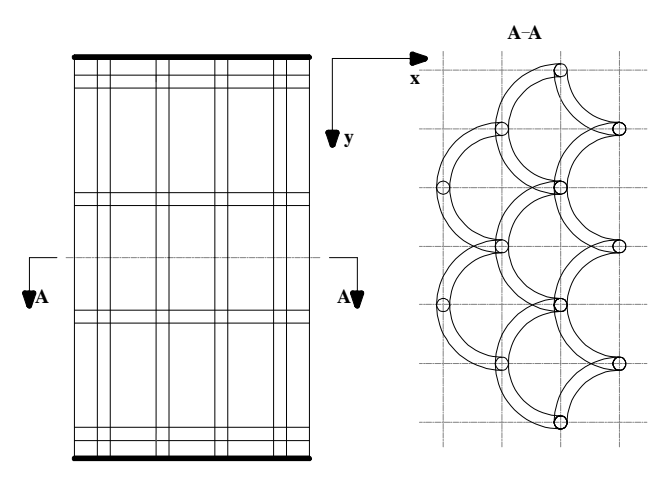

Fig. 1 Simplified structure for metal foams

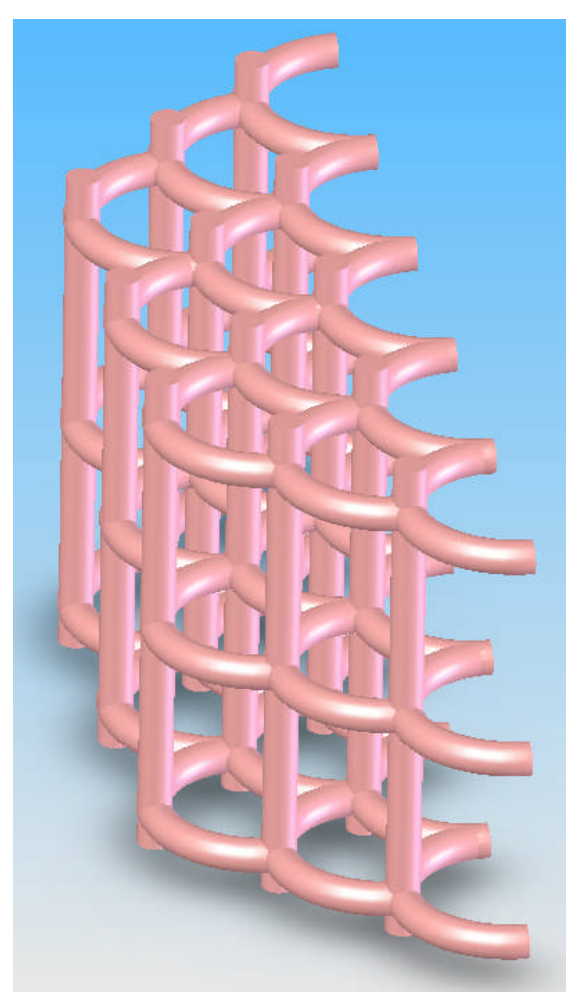

Fig. 23D schematic of the model for metal foams

Mo [4] developed a transport model based on the microstructure of metal foams, in which metal foams was simplified to two kinds of regular struts, called V-type and H-type. The cylinder diameter, specific surface area of metal foams and heat transfer coefficient had good agreement 
with previous studies. However, for the H-type strut, the angles and the lengths of two fins are not equal to the distance of V-type fins, which contradicts with isotropic characteristic of metal foams. Consequently, analytical model in Mo [4] needs to be improved. In this model, the H-type strut is composed of two circular arcs with a quarter of circular constant radian, as shown in Fig. 1. It was noted that the radius of the H-type strut arcs is the same with distance of two adjacent vertical fins. The three dimensional schematic for the microstructure of metal foams was shown in Fig. 2. The details of the V-type can be found in Mo [4].

Due to the distributions and sizes of microstructures, the numbers of V-strut and $\mathrm{H}$-strut can be easily obtained by using Eq. (1) and Eq. (2).

$$
\begin{aligned}
& \xi_{1}=\frac{\Delta x \cdot 1}{d_{p}{ }^{2}} \cdot \frac{D}{d_{p}} \\
& \xi_{2}=\frac{\Delta x \cdot 1}{d_{p}{ }^{2}} \cdot \frac{D}{d_{p}}
\end{aligned}
$$

For metal foams with fixed porosity and pore density, the fibre diameter of the solid matrix and the surface area density of the metal foams can be calculated as follows:

$$
\begin{gathered}
1-\varepsilon=\frac{\frac{1}{d_{p}{ }^{2}} \cdot \frac{D}{d_{p}} \cdot \Delta x \cdot 1 \cdot \frac{\pi}{4} d_{f}{ }^{2} d_{p}+\frac{1}{d_{p}{ }^{2}} \cdot \frac{D}{d_{p}} \cdot \Delta x \cdot 1 \cdot \frac{\pi}{4} d_{f}{ }^{2} \pi d_{p}}{D \cdot \Delta x \cdot 1} \\
d_{f}=\sqrt{\frac{4 \cdot(1-\varepsilon)}{\pi+\pi^{2}}} \cdot d_{p} \\
a_{s f}=\frac{\frac{1}{d_{p}{ }^{2}} \cdot \frac{D}{d_{p}} \cdot \Delta x \cdot 1 \cdot \pi d_{f} d_{p}+\frac{1}{d_{p}{ }^{2}} \cdot \frac{D}{d_{p}} \cdot \Delta x \cdot 1 \cdot \pi d_{f} \pi d_{p}}{D \cdot \Delta x \cdot 1}=\frac{\left(\pi+\pi^{2}\right) d_{f}}{d_{p}{ }^{2}}
\end{gathered}
$$

\section{Heat transfer model for flow boiling}




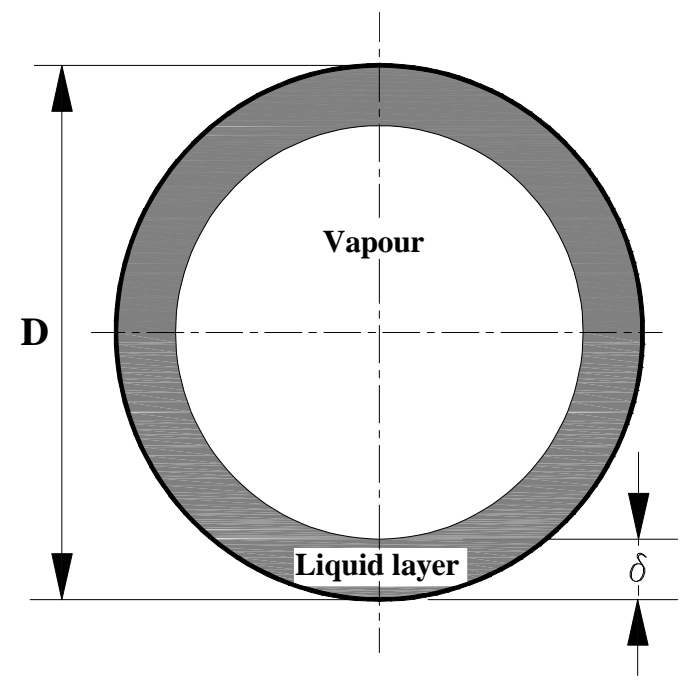

Fig. 3 Physical schematic for flow boiling in metal-foam filled tube

Flow boiling with annular flow pattern is considered, and is depicted in Fig. 3. It can be seen that the flow is divided into two regions, which are liquid layer near the heating wall and vapor zone contained in the liquid-vapor interface. Superposition correlation by Chen [3] has been adopted in this paper. It is assumed that nucleate boiling takes place in the liquid layer and equivalent flow boiling existed at the interface. Thus,

$$
h_{\text {equiv }}=h_{n b}+h_{\text {int } e r}
$$

Heat transfer coefficient for nucleate boiling was formulated by Tong and Tang [13] as follows:

$$
h_{n b}=0.00122 \cdot S \cdot\left(\frac{\lambda_{l}^{0.79} c_{p l}{ }^{0.45} \rho_{l}{ }^{0.49}}{\sigma^{0.5} \mu_{l}^{0.29} r^{0.24} \rho_{g}{ }^{0.49}}\right) \cdot\left(\Delta T_{\text {sat }}\right)^{0.24} \cdot\left(\Delta P_{\text {sat }}\right)^{0.75}
$$

For liquid phase, the pressure gradients is transferred as follows,

$$
\Delta P_{\text {sat }}=\rho_{l} \cdot R_{g} \cdot \Delta T_{\text {sat }}
$$

For this case,

$$
h_{n b}=0.00122 \cdot S \cdot\left(\frac{\lambda_{l}^{0.79} c_{p l}{ }^{0.45} \rho_{l}^{0.49}}{\sigma^{0.5} \mu_{l}^{0.29} r^{0.24} \rho_{g}{ }^{0.49}}\right) \cdot\left(\rho_{l} \cdot R_{g}\right)^{0.75} \cdot \Delta T_{s a t}
$$

With the intermediate variable $B$ being used,

$$
B=0.00122 \cdot S \cdot\left(\frac{\lambda_{l}^{0.79} c_{p l}{ }^{0.45} \rho_{l}^{0.49}}{\sigma^{0.5} \mu_{l}^{0.29} r^{0.24} \rho_{g}^{0.49}}\right) \cdot\left(\rho_{l} \cdot R_{g}\right)^{0.75}
$$

the equivalent heat transfer coefficient $h_{\text {equal }}$ can be simplified as follows: 


$$
\begin{gathered}
h_{\text {equal }}=h_{\text {int } e r}+\frac{B \cdot q}{h_{\text {inter }}} \\
h_{\text {equal }}=\frac{h_{\text {inter }}+\sqrt{h_{\text {inter }}^{2}+4 B \cdot q}}{2}
\end{gathered}
$$

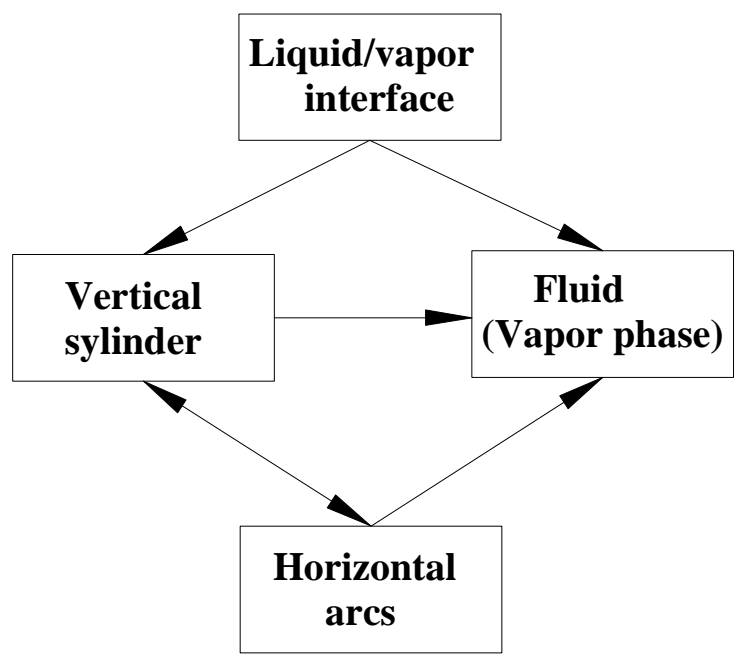

Fig. 4 Heat transfer network for flow boiling in metal foams

The model for flow boiling in metal-foam filled tube is based on fin analysis, and heat transfer mechanism is predicted by network of fluid and different types of simplified structures for metal foams in terms of effects on heat transfer performance. The heat transfer network is shown in Fig. 4. It indicates that convective heat transfer takes place between the flowing vapor and the liquid-vapor interface, between V-type strut and H-type strut of metal foams, respectively, and this affects the fraction and the thickness of liquid layer of two-phase flow in annular pattern. However, the temperature at the liquid-vapor interface is fixed at the saturated value due to coexistence of liquid phase and vapor phase. The heat transfer coefficient at the liquid-vapor interface for metal-foam tube could be analysed through investigating heat transfer performance of the vapor surrounded by annular liquid.

\section{Analysis of interface heat transfer for flow boiling in metal foams}

For the vertical cylinder, the simplified governing equation is shown in Eq. (13) as follows:

$$
\frac{\partial^{2} T}{\partial y^{2}}-\frac{4 h \cdot\left(T-T_{c}\right)}{d_{f} \cdot \lambda_{s}}=0
$$

Excess temperature is defined as:

$$
\theta=T-T_{c}
$$


With $m_{1}$ being introduced for simplicity consideration, the energy equation is obtained in Eq. (16).

$$
\begin{gathered}
m_{1}=\sqrt{\frac{4 h_{1}}{\lambda_{s} \cdot d_{f}}} \\
\frac{d^{2} \theta}{d y^{2}}-m_{1}^{2} \cdot \theta=0
\end{gathered}
$$

Eq. (17) and Eq. (18) give relevant boundary conditions as follows:

$$
\begin{aligned}
& \left.\theta\right|_{y=\delta}=\left.T\right|_{y=\delta}-T_{s a t} \\
& \left.\frac{\partial \theta}{\partial y}\right|_{y=D}=0
\end{aligned}
$$

By solving Eq. (16), the excess temperature distribution is obtained, which is shown in Eq. (19).

$$
\theta=\left.\theta\right|_{y=\delta} \cdot \frac{\operatorname{ch}\left[m_{1} \cdot(D-\delta-y)\right]}{\operatorname{ch}\left[m_{1}(D-\delta)\right]}
$$

Dimensionless temperature is defined as:

$$
\omega=\frac{\theta}{\left.\theta\right|_{y=\delta}}=\frac{\operatorname{ch}\left[m_{1} \cdot(D-\delta-y)\right]}{\operatorname{ch}\left[m_{1}(D-\delta)\right]}
$$

So the average temperature Is formulated in Eq. (21) and Eq. (22),

$$
\begin{aligned}
& \bar{\omega}=\frac{1}{D-\delta} \cdot \int_{0}^{D-\delta} \frac{\operatorname{ch}\left[m_{1} \cdot(D-\delta-y)\right]}{\operatorname{ch}\left[m_{1}(D-\delta)\right]} \cdot d y \\
& =\left.\frac{1}{D-\delta} \cdot \frac{1}{\operatorname{ch}\left[m_{1}(D-\delta)\right]} \cdot \frac{1}{m_{1}} \cdot \operatorname{ch}\left[m_{1}(D-\delta-y)\right]\right|_{0} ^{D-\delta} \\
& =\frac{\tanh \left[m_{1}(D-\delta)\right]}{m_{1}(D-\delta)} \\
& \bar{\theta}=\left.\theta\right|_{y=\delta} \cdot \bar{\omega}=\left.\theta\right|_{y=\delta} \cdot \frac{\tanh \left[m_{1}(D-\delta)\right]}{m_{1}(D-\delta)}
\end{aligned}
$$

According to the heat transfer network and the microstructure model for metal foams, the convective heat transfer coefficients between vapor and V-strut, between $\mathrm{H}$-strut of metal foams and liquid vapor interface can be easily obtained, and they are shown in Eq. (23) - Eq. (25),

$$
q_{1}=\pi d_{f} d_{p} h_{1} \cdot \bar{\theta}=\left.\pi d_{f} d_{p} h_{1} \cdot \theta\right|_{y=\delta} \cdot \frac{\tanh \left[m_{1}(D-\delta)\right]}{m_{1}(D-\delta)}
$$




$$
\begin{gathered}
q_{2}=\pi d_{f} \pi d_{p} h_{2} \cdot \bar{\theta}=\left.\pi d_{f} \pi d_{p} h_{2} \cdot \theta\right|_{y=\delta} \cdot \frac{\tanh \left[m_{1}(D-\delta)\right]}{m_{1}(D-\delta)} \\
q_{w}=\eta \cdot \Delta x \cdot h_{w} \cdot \theta(0)=\left.\eta \cdot \Delta x \cdot h_{w} \cdot \theta\right|_{y=\delta}
\end{gathered}
$$

$\eta$ denotes the ratio of bare wall surface area to the total wall surface area, and is given by Eq. (26).

$$
\eta=\frac{4}{a_{s f} \cdot D}
$$

From the Energy Conservation Law, the following equation can be obtained.

$$
\dot{m} \cdot C_{p} \cdot\left(T_{c}(x+\Delta x)-T_{c}(x)\right)=\xi_{1} \cdot q_{1}+\xi_{2} \cdot q_{2}+q_{w}
$$

By substituting Eq. (1) and Eq. (2) into Eq. (27), Eq. (28) is obtained.

$$
\begin{aligned}
& \dot{m} \cdot C_{p} \cdot\left(T_{c}(x+\Delta x)-T_{c}(x)\right)=\left.\frac{\Delta x \cdot 1}{d_{p}{ }^{2}} \cdot \frac{H}{d_{p}} \cdot \pi d_{f} d_{p} h_{1} \cdot \theta\right|_{y=\delta} \cdot \frac{\tanh \left[m_{1}(D-\delta)\right]}{m_{1}(D-\delta)}+ \\
& \left.\frac{\Delta x \cdot 1}{d_{p}{ }^{2}} \cdot \frac{H}{d_{p}} \cdot \pi d_{f} \pi d_{p} h_{2} \cdot \theta\right|_{y=\delta} \cdot \frac{\tanh \left[m_{1}(D-\delta)\right]}{m_{1}(D-\delta)}+\left.\frac{4}{a_{s f} \cdot H} \cdot \Delta x \cdot h_{w} \cdot \theta\right|_{y=\delta}
\end{aligned}
$$

The variable $l$ is defined as follows:

$$
\begin{aligned}
& l=\frac{1}{\dot{m} \cdot C_{p}}\left(\frac{1}{d_{p}^{2}} \cdot \frac{H}{d_{p}} \cdot \pi d_{f} d_{p} h_{1} \cdot \frac{\tanh \left[m_{1}(D-\delta)\right]}{m_{1}(D-\delta)}+\right. \\
& \left.\frac{1}{d_{p}^{2}} \cdot \frac{H}{d_{p}} \cdot \pi d_{f} \pi d_{p} h_{2} \cdot \frac{\tanh \left[m_{1}(D-\delta)\right]}{m_{1}(D-\delta)}+\eta \cdot h_{w}\right)
\end{aligned}
$$

Then

$$
T_{c}(x+\Delta x)-T_{c}(x)=\left.l \cdot \theta\right|_{y=\delta} \cdot \Delta x
$$

The temperature of the vapor phase was solved as follows:

$$
T_{c}(x)=\left(T_{i n}-\left.T\right|_{y=\delta}(x)\right) \cdot e^{-l x}+\left.T\right|_{y=\delta}(x)
$$

Therefore, the temperature at the liquid-vapor interface is obtained, which is shown in Eq. (32) and Eq. (33).

$$
\begin{gathered}
\left.T\right|_{y=\delta}(x)=\frac{T_{c}(x)-T_{i n} \cdot e^{-l x}}{1-e^{-l x}} \\
\left.\theta\right|_{y=\delta}=\left.T\right|_{y=\delta}(x)-T_{c}(x)=\left(\left.T\right|_{y=\delta}(x)-T_{i n}\right) \cdot e^{-l x}
\end{gathered}
$$


For vapour phase, convective heat flux is calculated in Eq. (34) and Eq. (35).

$$
\begin{aligned}
& Q(x)=\dot{m} C_{p}\left(\left.T\right|_{y=\delta}-T_{i n}\right) \cdot\left(1-e^{-l x}\right) \\
& Q(L)=\dot{m} C_{p}\left(\left.T\right|_{y=\delta}-T_{i n}\right) \cdot\left(1-e^{-l L}\right)
\end{aligned}
$$

In this way, the temperature difference between the interface and the averaged vapor phase is obtained in Eq. (36) as follows:

$$
\left.T\right|_{y=\delta}-\bar{T}_{c}=\frac{1-e^{-l L}}{l L}\left(\left.T\right|_{y=\delta}-T_{i n}\right)
$$

The heat transfer coefficient at the liquid-vapor interface is also formulated, which is shown in Eq. (37) and Eq. (38),

$$
\begin{array}{r}
h_{\text {inter }}=\frac{Q(L)}{A_{w}\left(\left.T\right|_{y=\delta}-\overline{T_{c}}\right)}=\frac{\dot{m} C_{p}\left(\left.T\right|_{y=\delta}-T_{0}\right) \cdot\left(1-e^{-l L}\right)}{1 \cdot L \frac{1-e^{-l L}}{l L}\left(\left.T\right|_{y=\delta}-T_{0}\right)}=\dot{m} C_{p} l \\
h_{\text {int } e r}=\frac{1}{d_{p}{ }^{2}} \cdot \frac{(H-\delta)}{d_{p}} \cdot \pi d_{f} d_{p} h_{1} \cdot \frac{\tanh \left(m_{1}(H-\delta)\right)}{m_{1}(H-\delta)}+ \\
\frac{1}{d_{p}{ }^{2}} \cdot \frac{(H-\delta)}{d_{p}} \cdot \pi d_{f} \pi d_{p} h_{2} \cdot \frac{\tanh \left(m_{2}(H-\delta)\right)}{m_{2}(H-\delta)}+\eta \cdot h_{w}
\end{array}
$$

\begin{tabular}{|c|c|c|}
\hline Vertical struts & Horizontal struts & Bare wall \\
\hline $\bar{N} u_{d}=1.13 \cdot C_{1} \cdot R e_{d, \max }{ }^{m} \cdot \operatorname{Pr}^{1 / 3}$ & $\bar{N} u_{d}=1.13 \cdot C_{1} \cdot R e_{d, \max }{ }^{m} \cdot \operatorname{Pr}^{1 / 3}$ & $N u=0.021 \cdot \operatorname{Re}^{0.8} \cdot \operatorname{Pr}^{0.5}$ \\
\hline$R e_{d, \max }=\frac{\rho \cdot d_{f} \cdot V_{v, \text { max }}}{\mu_{f}}$ & $R e_{d, \text { max }}=\frac{\rho \cdot d_{f} \cdot V_{v, \text { max }}}{\mu_{f}}$ & \\
\hline$V_{v, \max }=\frac{d_{p}}{d_{p}-d_{f}} V_{i n}$ & $V_{h, \max }=\frac{\frac{d_{p}}{2}}{d_{p} \cdot \pi / 2-d_{f}} V_{i n}$ & \\
\hline
\end{tabular}

Here $h_{1}, h_{2}$ and $h_{w}$ represent heat transfer coefficients on vertical strut, horizontal strut and bare wall respectively [4], and the details are listed in Table 1 and Table 2.

Table 1 Correlations of convective heat transfer coefficients across single cylinder

Table 2 The values of the constants shown in Nusselt number correlations 


\begin{tabular}{ccc}
$R e_{d, \max }$ & $C_{1}$ & $m$ \\
$0.4-4$ & 0.989 & 0.330 \\
$4-40$ & 0.911 & 0.385 \\
$40-4000$ & 0.683 & 0.466 \\
$4000-40000$ & 0.193 & 0.618 \\
$40000-400000$ & 0.027 & 0.805 \\
\hline
\end{tabular}

\section{Results and discussions}

The model has been verified by comparison with previous investigations on flow boiling in metal foams filled tube, as shown from Fig. 5 to Fig. 7. In Fig. 5 that the equivalent boiling heat transfer coefficients under different mass flow rates agree mainly with the experimental results by Zhao et al. [10]. The analytical results are a little higher than the experimental data with a maximum deviation of $25 \%$. However, for fixed fibre diameter of metal foams, the results predicted by the model agree well with the experimental data, and this is shown in Fig. 6. The case for the high density heat flux is also investigated for comparison, which is shown in Fig. 7.

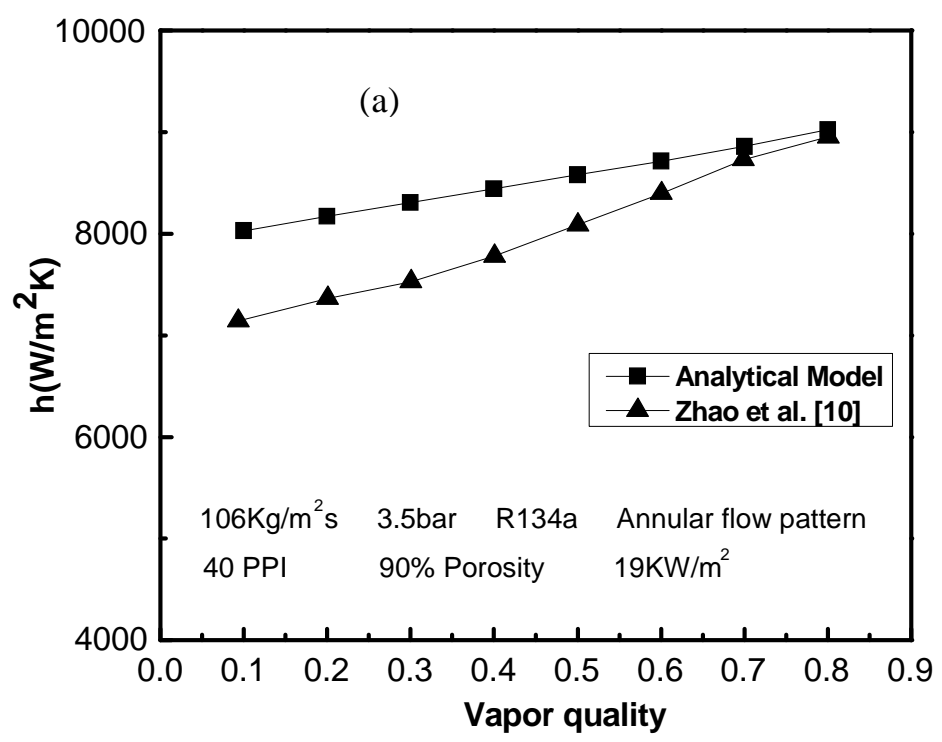




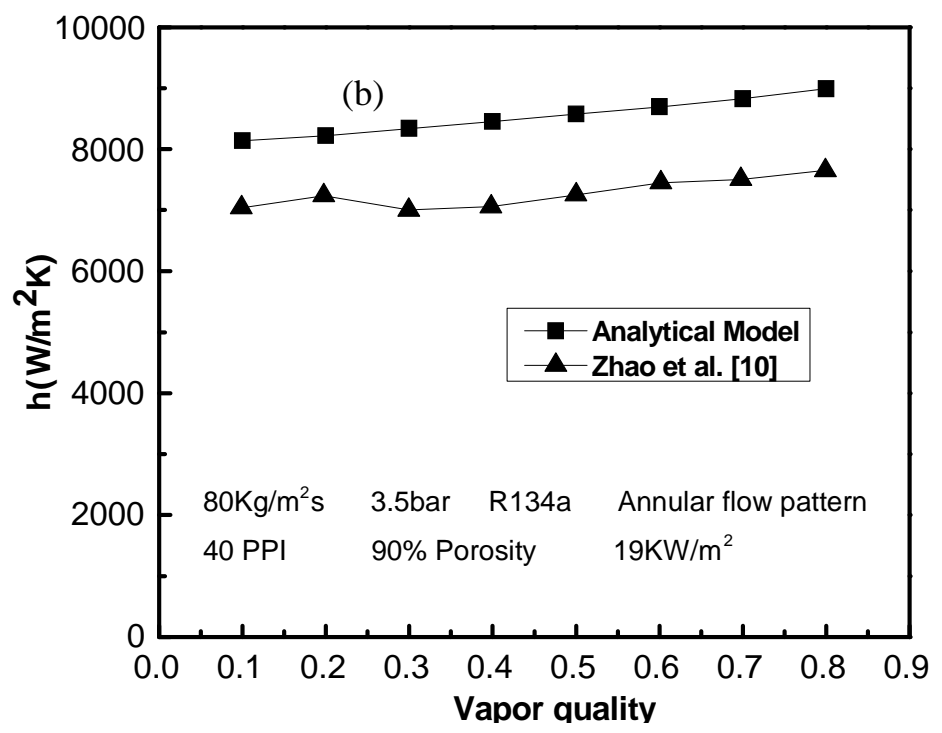

Fig. 5 Validation of the model under different mass flow rate

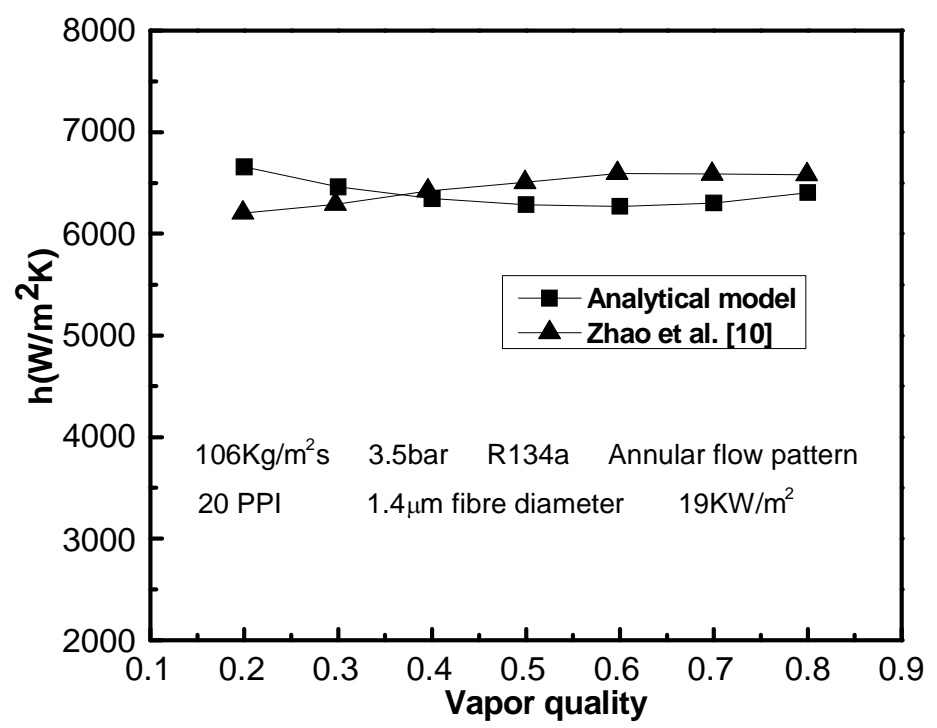

Fig. 6 Comparison of heat transfer coefficient at fixed fibre diameter 


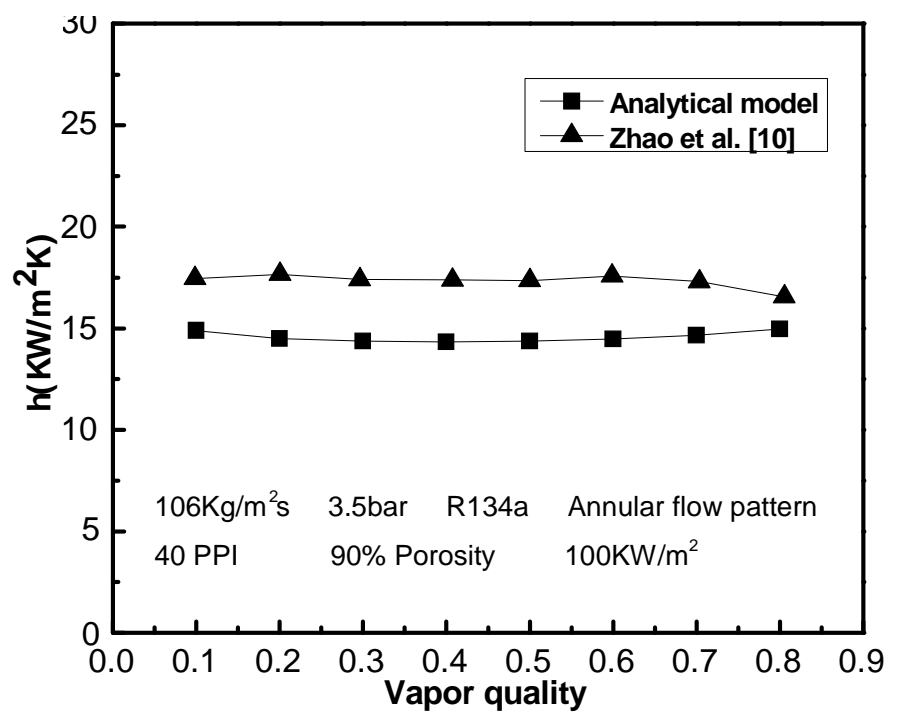

Fig. 7 Comparison of heat transfer coefficients for high heat flux

$\beta$ denotes the ratio of nucleate boiling to the whole heat transfer coefficient, given by Eq. (34).

$$
\beta=\frac{h_{N B}}{h_{\text {equiv }}}
$$

The distribution of $\beta$ is shown in Fig. 8. It was obvious that the ratio decreases with vapor quality increasing for refrigerant R134a in the annular flow pattern. It can be attributed to the reduction of liquid layer thickness nearby the heated wall. It also indicates that proportion of nucleate boiling reaches the minimum value when vapor quality equals 0.7 .

Fig. 9 is the effect of heat flux at the wall on the ratio of nucleate boiling. It is seen that the ratio goes up with the increase of heat flux. The average value of the ratio is larger than 0.5 , which indicates the dominant role of the nucleate boiling.

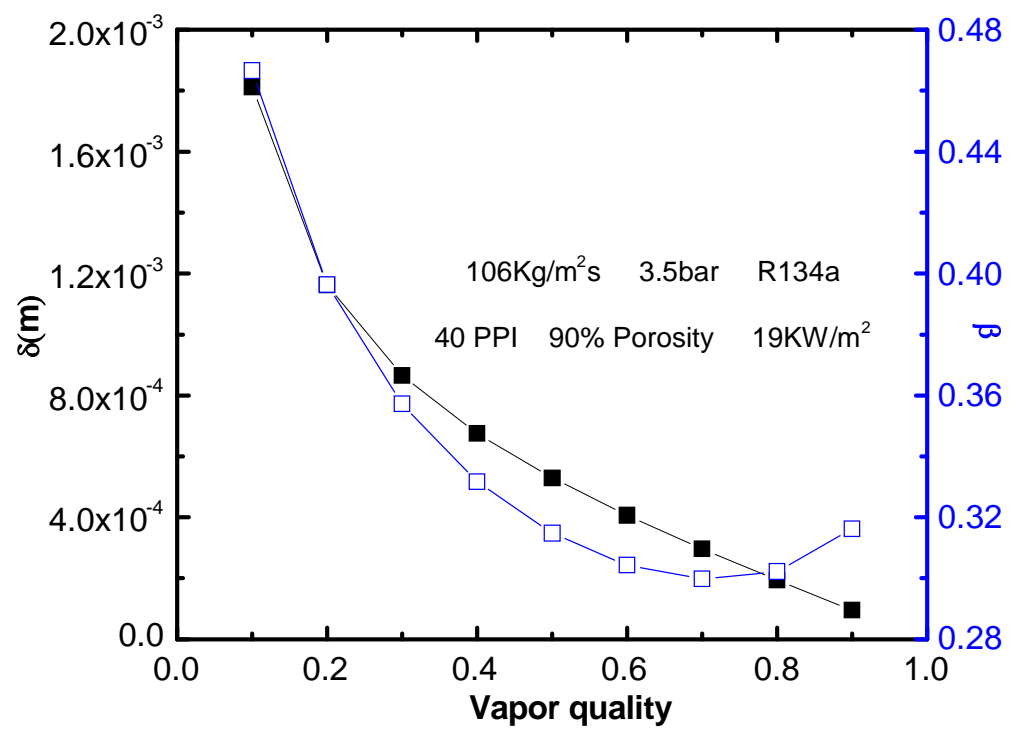


Fig. 8 Composition of heat transfer for flow boiling

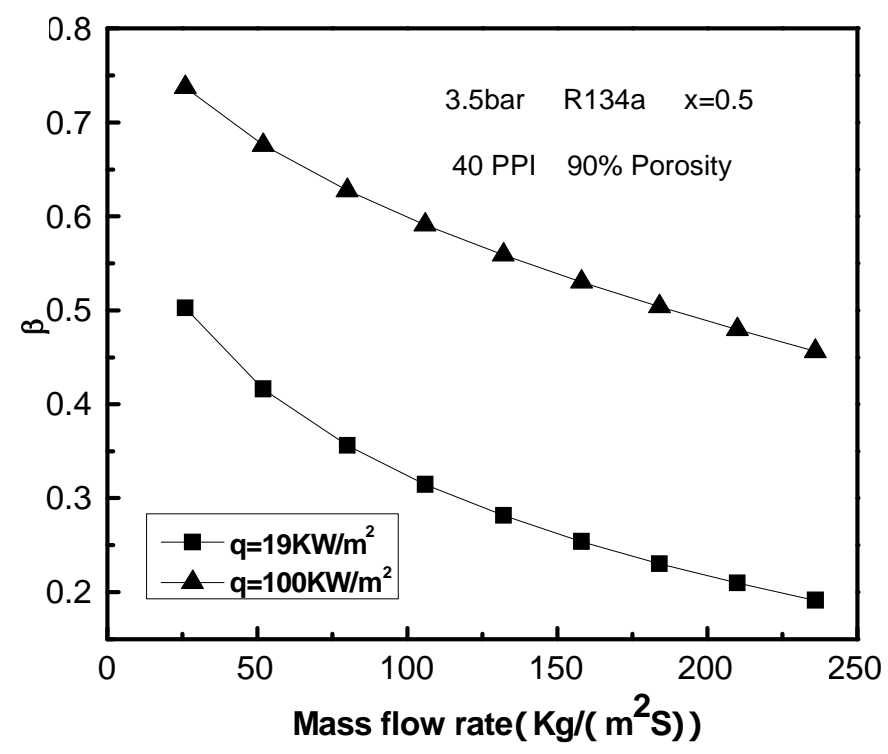

Fig. 9 Effect of heat flux on nucleate boiling

The effect of microstructure of metal foams on heat transfer is analysed in Fig. 10. From the analysis above, convective boiling heat transfer is influenced by the fibre diameter and pore diameter of the metal matrix. Fig. 10 shows that heat transfer of equivalent convective boiling and forced convection is enhanced with the increase of $d_{f} / d_{p}{ }^{2}$. In addition, the differences of the heat transfer coefficients between these two cases in Fig. 10 become smaller when $d_{f} / d_{p}{ }^{2}$ increases, indicating that forced convection plays more dominant role with increasing $d_{f} / d_{p}{ }^{2}$.

Saturated pressure of refrigerant R134a is also investigated due to its influence on property of liquid phase and vapor phase, and it is shown by Fig. 11 that the influence of saturated pressure is limited under annular flow pattern for refrigerant R134a. 


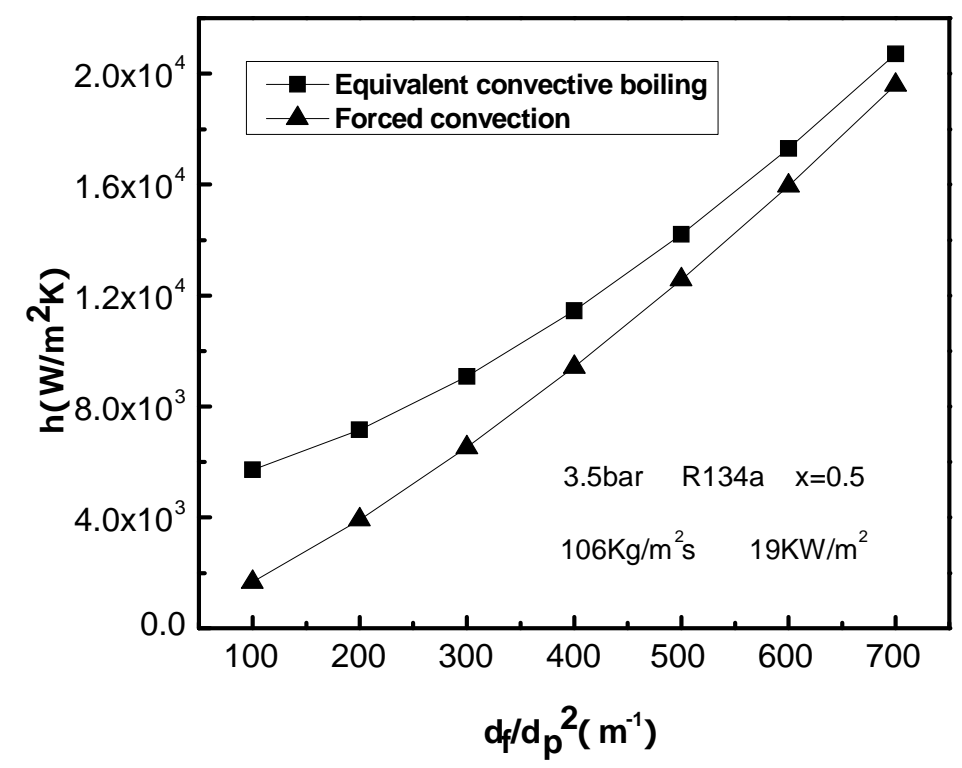

Fig. 10 Effect of metal foams on heat transfer performance

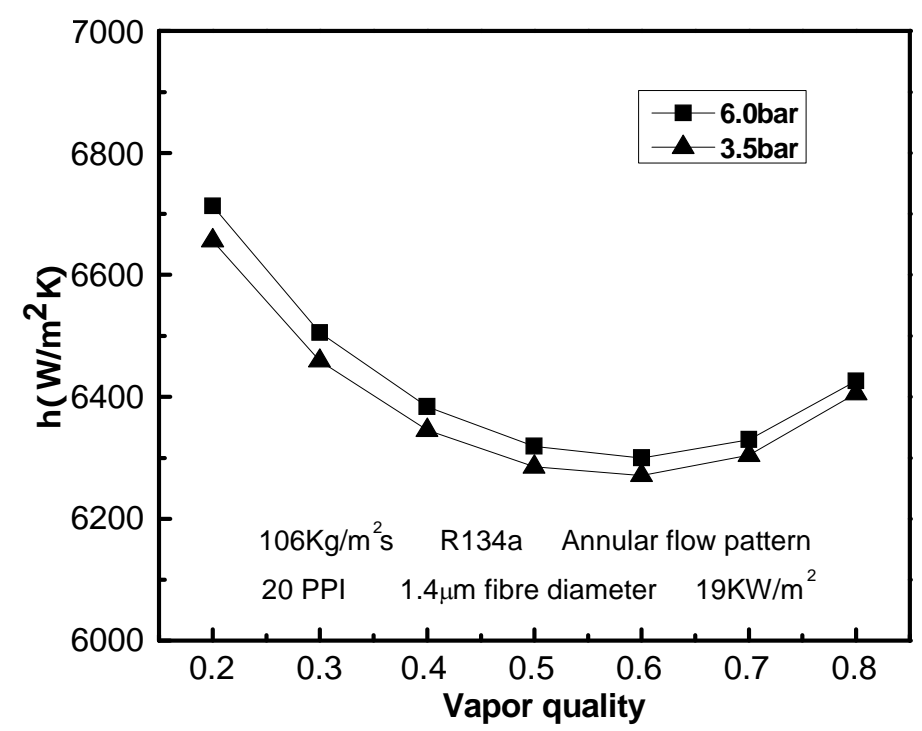

Fig. 11 Effect of saturated pressure on heat transfer performance

\section{Conclusions}

A newly simplified microstructure model of metal foams has been developed, and the equivalent heat transfer coefficient for flow boiling in metal-foam filled tube is analytically studied based on the microstructure model and fin analysis method. It is found that the fibre diameter, surface area density of metal foams and heat transfer coefficient predicted by analytical approach show quite good agreement with experimental data by other researchers. The weight of nucleate boiling is reduced with the increase of vapor quality and the decrease of the heat flux at the wall. The equivalent heat transfer coefficients of flow boiling and forced convection can be increased with increasing $d_{f} / d_{p}{ }^{2}$, whilst the influence of saturated pressure is limited under annular flow pattern for refrigerant R134a. 
Acknowledgements This work is supported by the National Basic Research Program (2006CB601203), the National Natural Science Foundation of China (50806057) and the Engineering and Physical Sciences Research Council (No: EP/F061439/1) .

\section{References}

1. Rohsenow WM (1953) Heat Transfer with Evaporation. In: Chapter Heat Transfer, A Symposium Held at the University of Michigan, University of Michigan Press, pp. 101-150

2. Dengler CE, Addoms JN (1956) Heat Transfer Mechanism for Vaporization of Water in a Vertical Tube. AIChE Chem Eng Progress Symp Ser 52: 95-103

3. Chen JC (1966) A Correlation for Boiling Heat Transfer to Saturated Fluids in Convective Flow. Ind Eng Chem Process Des Dev 5: 322-329

4. Mo B (2007) Numerical Evaluation of Heat Transfer and Pressure Drop in Open-cell Foams. M.S Thesis, University of Florida

5. Krishnan SM, Murthy JY, Garimella SV (2006) Direct simulation of Transport in Open-cell Metal Foam. ASME J Heat Transf 128: 793-799

6. Krishnan SM, Garimella SV, Murthy JY (2008) Simulation of Thermal Transport in Open-cell Metal Foams: Effect of Periodic Unit Cell Structure. ASME J Heat Transf 130: 024503-1-5

7. Boomsma K, Poulikakos D, Ventikos Y (2003) Simulation of Flow through Open Cell Metal Foams Using an Idealized Periodic Cell Structure. Int J Heat Fluid Flow 24: 825-834

8. Murthy S, Joshi Y, Gurrum S, Nakayama W (2006) Enhanced Boiling Heat Transfer Simulation from Structured Surfaced: Semi-analytical Model. Int J Heat Mass Transf 49: 1885-1895

9. Wang CY, Cheng P (1997) Multiphase flow and heat transfer in porous media. Adv Heat Transf 30: 93-196

10. Zhao C Y, Lu W and S A Tassou, (2009) Flow boiling heat transfer in horizontal metal foam tubes", ASME J. of Heat Transfer, 131:121002-1-8.

11. Lu W, Zhao CY (2009) Numerical Modeling of Flow Boiling Heat Transfer in Horizontal Metal-foam Tubes. Adv Eng Mater 11: 832-836

12. Pastuszko R, Poniewski ME (2008) Semi-analytical Approach to Boiling Heat Fluxes Calculation in Subsurface Horizontal and Vertical Tunnels. Int J Therm Sci 47: 1169-1183

13. Tong LS, Tang YS (1997) Boiling Heat Transfer and Two-phase Flow. The $2^{\text {nd }}$ edition, Series in Chemical and Mechanical Engineering, Taylor \& Francis Publishers, 1997 